\title{
Brief Introduction of Promoting the Chinese Program For Exploring the Martian System
}

\author{
Jinsong Ping ${ }^{1}$, Xian $\mathrm{Shi}^{2}$, Nianchuan $\mathrm{Jian}^{2}$, Sujun Zhang ${ }^{2}$, \\ Mingyuan Wang ${ }^{1}$, Kun Shang ${ }^{1}$ and Yinghuo-1 VLBI team ${ }^{1}$ \\ ${ }^{1}$ National Astronomical Observatory of China, CAS \\ email: pjs@shao.ac.cn \\ ${ }^{2}$ Shanghai Astronomical Observatory, CAS
}

\begin{abstract}
Following the progress of Chinese deep space exploration step, since 2006 we started a Mars mission, Yinghuo-1, by join in the Phobos-Grunt mission of Russia. A satellite bus platform and onboard payloads as well as an innovative open-loop radio tracking system have been developed by mission team. Also, together with Russian and German colleagues, we developed a kind of in-beam tracking method for measuring the rotation and nutation of Phobos, and developed the 1st Phobos global gravity field for the mission. We are promoting the Chinese new mission for Mars exploration. Although the joint YH-1 \& Phobos-Grunt mission failed, the new techniques and knowledge developed by mission teams may benifit the future missions. In fact, the open-loop technique have been applied into lunar and other planetary missions, and the method in developing Phobos global gravity field will be used in the study of Rosetta mission and future Chinese mission for small body.
\end{abstract}

